\title{
Glutationa S-transferase M1 (GSTM-1): distribuição étnica e relação com câncer
}

\author{
Glutathione S-transferase M1 (GSTM-1): ethnic distribution and relation with cancer
}

\author{
Roberta Losi-Guembarovski ${ }^{1}$; llce Mara de Syllos Cólus²
}

\begin{abstract}
Resumo: Os seres humanos apresentam diferenças individuais quanto ao risco de desenvolver câncer. Tais diferenças são provenientes, entre outros fatores, da capacidade geneticamente determinada dos organismos, desde bactérias até o homem, em ativar e detoxificar os carcinógenos. Assim sendo, a associação entre alelos específicos de genes responsáveis pela metabolização de compostos químicos e o risco aumentado ao desenvolvimento de tumores, se deve à existência de múltiplos passos enzimáticos no metabolismo, que podem resultar na ativação ou detoxificação de xenobióticos. O maior grupo de genes de detoxificação já descritos até o momento envolve a grande família das enzimas Glutationa S-transferases (GSTs). O gene GSTM-1, que faz parte desta família, é polimórfico na população e ocorre em cerca de $30-50 \%$ dos indivíduos, dependendo do grupo étnico a que pertencem. Em diversos trabalhos descritos na literatura, o gene GSTM-1 vem sendo freqüentemente associado a um risco elevado de desenvolvimento de diversos tipos de tumores, principalmente o câncer de pulmão, o que torna de grande importância a determinação da freqüência deste gene nas diferentes populações, buscando a identificação de marcadores de susceptibilidade ao câncer.
\end{abstract}

Palavras-chave: GSTM-1; câncer; grupos étnicos.

\begin{abstract}
Interindividual differences concerning the risk of cancer development are mostly due to a genetically determined capacity of the organism, from bacteria to man, in activating and detoxifying carcinogens. Therefore, the association among specific alleles of enzymes that are able to metabolize chemical compounds and the risk of developing several cancers, is attributed to the existence of various enzymatic steps in biometabolism, which can result in the activation or detoxification of xenobiotics. A great part of detoxification genes which have already described, is related to Glutathione S- transferase (GSTs) enzyme family. GSTM-1 gene, which belongs to this family, is polimorfic in the population and occurs in about $30-50 \%$ of the individuals, depending on the ethnical group which they belong to. In several works described in literature, GSTM-1 gene has been frequently associated to a high risk of developing several types of cancer, mostly lung cancer. Such informations are extremely important in order to determine the frequency of this gene in different populations, searching for an identification of markers that indicate cancer susceptibility.
\end{abstract}

Key words: GSTM-1 gene; cancer; ethnics groups.

\section{Introdução}

Estudos epidemiológicos têm demonstrado que mais de $90 \%$ de todos os cânceres estão relacionados a fatores ambientais, como o tabaco e a dieta, e que diversos compostos químicos parecem interagir com as macromoléculas celulares e iniciar o processo tumoral (RAUNIO et al., 1995).

Devido ao fato de que praticamente todos os organismos vivos estão continuamente expostos a compostos químicos, vários mecanismos de proteção foram desenvolvidos durante o processo evolutivo, sendo a metabolização a mais versátil destas formas de proteção. Assim, os animais mais evoluídos como o homem, desenvolveram no fígado um mecanismo enzimático que intercepta os xenobióticos, como por exemplo a nicotina, transformando-os em substâncias mais hidrossolúveis, facilmente excretadas do organismo (KALANT, 1991), impedindo seu acúmulo e, conseqüentemente, o surgimento de danos no material genético.
A biotransformação das drogas, em especial as que se processam no fígado, são comumente agrupadas em duas fases: 1 e 2. Na fase 1, geralmente realizada pelas enzimas da superfamília do Citocromo P-450 (CYP), que inclui as reações de oxidação, de redução ou de hidrólise, as drogas podem ser ativadas, inativadas ou terem inalteradas suas atividades. Durante a fase 2, que envolve reações de conjugação, quase sempre ocorre a inativação total do xenobiótico, caso este ainda não tenha sido inativado na fase 1, o que é geralmente realizado pelas enzimas da família das Glutationa S-transferases (GSTs) e N-acetiltransferases (NATs). No entanto, quando os produtos formados na fase 1 não sofrem inativação ou são ativados a substâncias mais reativas pelas enzimas da fase 2 , estes intermediários reativos podem se ligar covalentemente ao DNA e causar diversas formas de dano ao organismo, pois agirão como agentes mutagênicos e/ou carcinogênicos (PARKE, 1987; NEBERT, 1991; KALANT, 1991; VENITT, 1994; RAUNIO et al., 1995) (Figura 1).

\footnotetext{
${ }^{1}$ Mestre em Genética pela Universidade Estadual de Londrina (UEL).

${ }^{2}$ Docente do Departamento de Biologia Geral da UEL.
} 


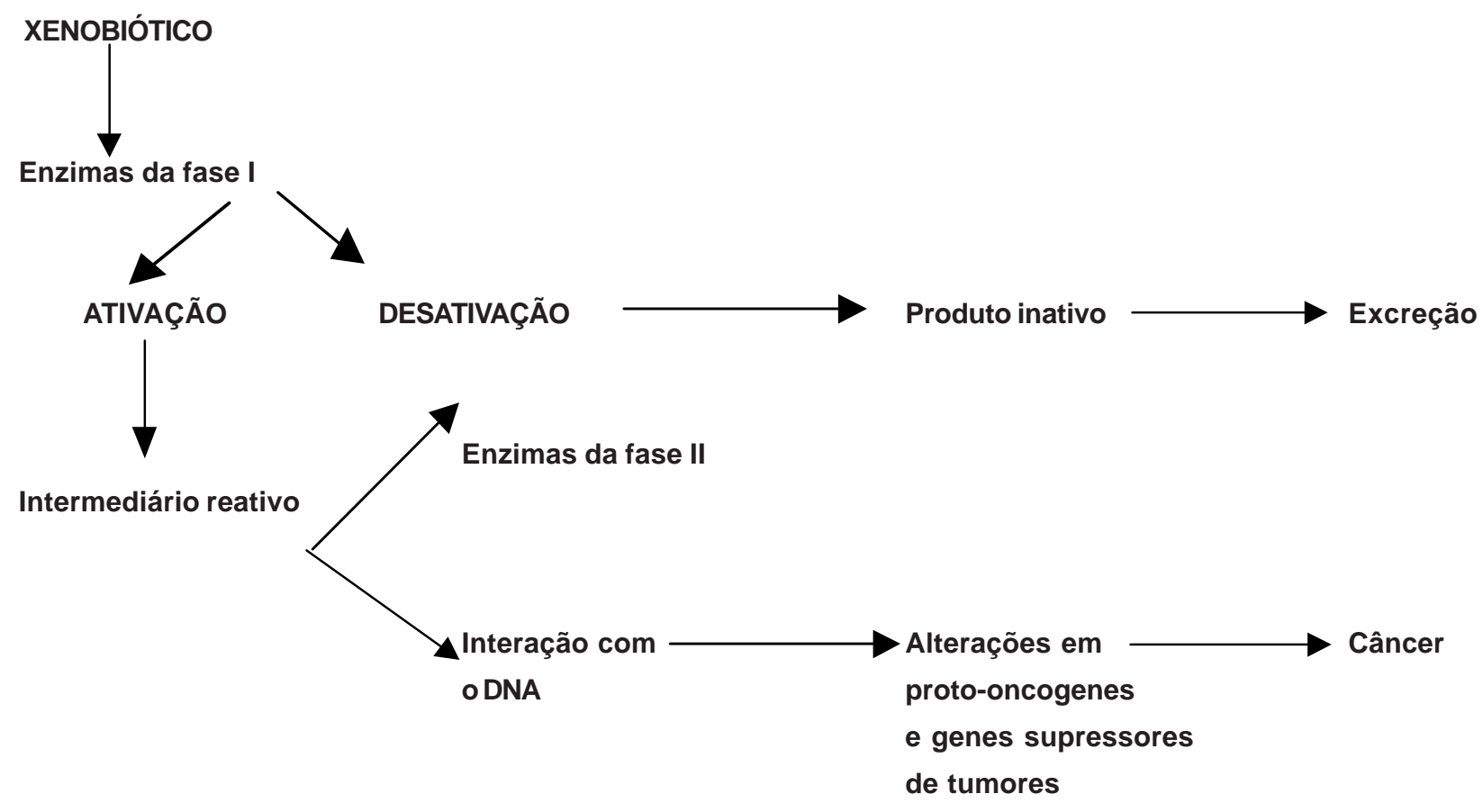

Figura 1 - Seqüência dos efeitos biológicos nas reações de biotransformação dos xenobióticos.

Os genes que codificam as enzimas das fases 1 e 2 vêm sendo clonados e identificados em seres humanos e muitos deles mostram-se polimórficos dentro da população, parecendo contribuir para a susceptibilidade individual ao desenvolvimento de tumores (NEBERT, 1991; VESELL, 1991; SHIELDS, 1994; HIRVONEN, 1995; RAUNIO et al., 1995). A associação entre defeitos nas enzimas CYPs, GSTs e NATs com o aumento do risco de desenvolvimento de câncer de pulmão, bexiga, bem como de outros tipos de cânceres em indivíduos fumantes parece confirmar esta hipótese (ZHONG et al., 1993a; RAUNIO et al., 1995).

Assim, entre os mecanismos que estão envolvidos no processo de carcinogênese, podemos destacar as alterações em genes responsáveis pela metabolização de xenobióticos (CAVENEE; WHITE, 1995), de maneira que distúrbios no balanço entre ativação e detoxificação podem, então, explicar as variações individuais na resposta à exposição a carcinógenos (HIRVONEN, 1995).

\section{Gene GSTM-1 e Câncer}

Dentre os sistemas de detoxificação já descritos, as GSTs possuem um importante papel, fornecendo proteção contra compostos eletrofílicos e produtos do estresse oxidativo, sugerindo que estas enzimas fazem parte de um mecanismo de resposta adaptativa aos danos causados por compostos químicos (HAYES; PULFORD, 1995). Esta classe de enzimas é de grande importância na manutenção da integridade do genoma celular e, como resultado, possui um importante papel na susceptibilidade individual ao câncer (GESLEIR; OLSHAN, 2001).
As GSTs estão amplamente distribuídas na natureza, encontradas desde a bactéria até o homem, e são expressas em tecidos de mamíferos (HAYES; PULFORD, 1995). Segundo De Jong et al. (1991), as GSTs humanas são produtos de uma família de genes localizados em diferentes cromossomos humanos, que consistem de 5 classes: alfa, $\mathrm{m} \mu$, pi, theta, que são encontradas no citossol e uma microssomal. Dentre estas, a classe $\mu$ é talvez a mais extensa, possuindo 4 isoenzimas, GSTM-1, GSTM-2, GSTM-3 e GSTM-4.

O gene GSTM-1 foi mapeado no cromossomo 1 (DEJONG et al., 1991), na região 1p13 (ZHONG et al., 1992; PEARSON et al., 1993; ROSS et al., 1993). Este gene é polimórfico, apresentando os alelos GSTM-1a e GSTM-1b, que diferem entre si por uma substituição em um único par de bases (COTTON et al., 1999). O genótipo GSTM-1 nulo possui uma deleção no gene que codifica GSTM- $1 \mathrm{a} / \mathrm{b}$. Os alelos podem se combinar formando genótipos heterozigotos (GSTM-1ab, GSTM1b_e GSTM-1a_) e homozigotos (GSTM-1aa, GSTM1 bb_ou GSTM-1_). Em indivíduos que possuem o genótipo homozigoto nulo para GSTM-1, a atividade desta enzima está ausente e não há expressão nem de GSTM-1a, nem de GSTM-1b. (FRYER et al., 1993; PEARSON et al., 1993; ZHONG et al, 1993b). Desta maneira, a ausência deste gene pode causar um maior acúmulo de metabólitos reativos no organismo, aumentando a probabilidade de interação com as macromoléculas celulares e iniciação do processo tumoral.

O genótipo GSTM-1 nulo já foi associado ao desenvolvimento de diversos tipos de cânceres, como o de pulmão (HIRVONEN et al. 1993); coloretal (ZHONG et al., 1993a; DEAKIN et al., 1996); bexiga (LIN et al., 1994; SANTELLA et al., 1995; JOHNS; HOULSTON 
2000); cólon e mama (MCWILLIAMS et al., 1995); adenocarcinoma de cólon e estômago (STRANGE et al., 1991) e carcinoma de cavidade oral (SATO et al., 1999).

Dentre os tipos de tumores freqüentemente associados à ausência do gene GSTM-1, destaca-se o câncer de pulmão, pelo fato de que GSTM-1a e GSTM-1b são ativos na detoxificação de certos epóxidos dos hidrocarbonetos aromáticos policíclicos
(PAH) encontrados na fumaça do cigarro e em outros produtos de combustão (HAYES; PULFORD, 1995). Assim sendo, hidrocarbonetos aromáticos policíclicos presentes no cigarro, incluindo o carcinógeno benzo(a)pireno, requerem primariamente ativação metabólica pelas enzimas da fase 2 (citocromo P450 e suas formas ativadas) que produzem um intermediário que se constitui no substrato de detoxificação para as enzimas GSTM-1 da fase 2 (Figura 2).

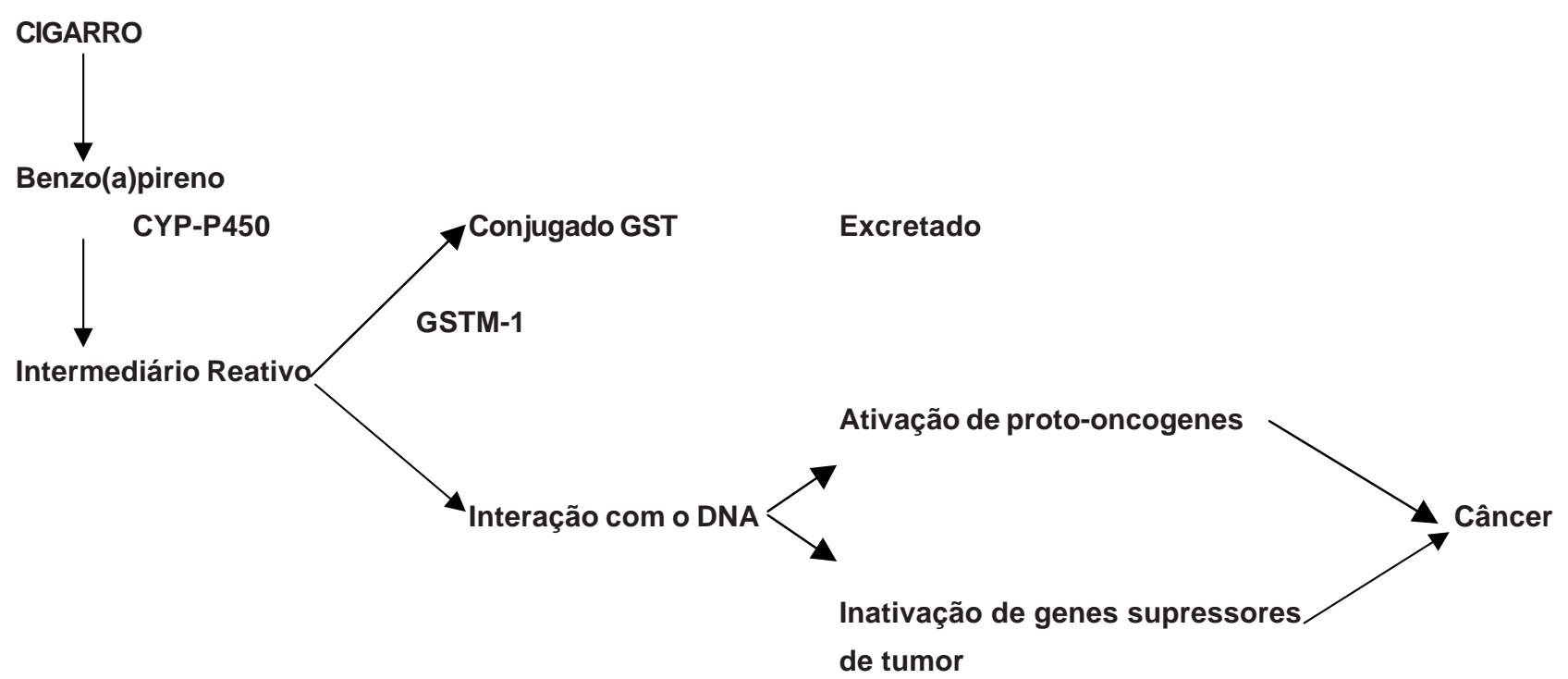

Figura 2 - Biotransformação dos produtos do cigarro pelas enzimas CYP-P450 e GSTM-1 e relação com o câncer

O risco de desenvolvimento de câncer de pulmão, portanto, se torna dramaticamente mais alto naqueles indivíduos que possuem simultaneamente genótipos de alto risco para CYP e GST (RAUNIO et al., 1995). Desta maneira, a capacidade de detoxificar os PAH é limitada em indivíduos onde falta a enzima GSTM-1, e a susceptibilidade genética ao câncer de pulmão, entre outros, pode depender do balanço metabólico entre as enzimas das fases 1 e 2 (HAYASHI et al., 1992; VAN POPPEE et al., 1992 e NAKAGIMA et al., 1995).

Diversos estudos mostram uma alta correlação entre o hábito tabagista e o desenvolvimento de câncer de pulmão em indivíduos que possuem o genótipo GSTM-1 nulo. Alguns destes indicam também uma relação entre a ausência deste gene e o aumento do número de aductos no DNA, além de uma deficiência na detoxificação de carcinógenos presentes no tabaco (HARADA et al., 1987; SEIDEGARD et al., 1990; VAN POPPEL et al., 1992; SHIELDS et al., 1993; NAKAGIMA et al., 1995; KIHARA et al., 1995 e FORD et al., 2000).

Existem estudos que não encontraram uma correlação positiva entre a ausência do gene GSTM-1 e câncer de pulmão (KETTERER et al., 1992; ALEXANDRIE et al., 1994; LONDON et al., 1995; RYBERG et al., 1997; FIGUEIRAS et al., 1997 e BUIM, 1999) e esôfago (BUIM, 1999). Gesleir e Olshan (2001) também não encontraram uma associação positiva entre o genótipo GSTM-1 nulo e o desenvolvimento de tumores de cabeça e pescoço.

A falta de correlação entre o genótipo de susceptibilidade para o gene GSTM-1 e o câncer, segundo Hayes e Pulford (1995), pode ser conseqüência de múltiplos fatores, como o uso incorreto ou insuficiente de grupos controles, variações no hábito tabagista, nas dietas alimentares dos indivíduos e a presença de outros fatores ambientais.

Apesar de alguns trabalhos não demonstrarem uma associação positiva entre o genótipo GSTM-1 nulo e o desenvolvimento de câncer, Conforti-Froes et al. (1997) acreditam que a predisposição genética relacionada ao gene GSTM-1 pode ser um dos principais mecanismos envolvidos na carcinogênese pulmonar, podendo ser bastante útil como biomarcador para este tipo de tumor em indivíduos fumantes.

Já está bem estabelecido que a distribuição polimórfica das enzimas metabolizadoras nas diferentes populações ao redor do mundo influencia na susceptibilidade ao desenvolvimento de doenças ambientais, sendo de grande importância o conhecimento da freqüência destes genes nas diferentes etnias, visando gerar uma forma efetiva de prevenção, especialmente contra o câncer (AU et al., 1999). 


\section{Gene GSTM-1 e Variações étnicas}

Diferentes grupos étnicos ao redor do planeta apresentam diferenças em relação à presença ou ausência do gene GSTM-1 e, de acordo com Lin et al. (1994), como tais variações étnicas podem influenciar o poder e a interpretação dos resultados de estudos epidemiológicos, existe uma grande necessidade em se estudar o polimorfismo deste gene nas diferentes etnias.

O gene GSTM-1 encontra-se ausente em cerca de $30-50 \%$ dos indivíduos, de acordo com a etnia a que estes pertencem. Uma avaliação do polimorfismo do gene GSTM-1 em indivíduos saudáveis mostrou que o genótipo nulo ocorre em cerca de 23 a $41 \%$ de afroamericanos e negros; 23 a $48 \%$ em populações africanas; 33 a $63 \%$ em populações asiáticas e $57 \%$ de caucasianos (LONDON et al., 1995; COTTON et al., 1999; CHEN et al., 1999). McWilliams et al. (1995), revisando trabalhos que estudaram grupos-controle para o polimorfismo do gene GSTM-1, concluíram que a ausência do gene ocorre em cerca de $47 \%$ da população japonesa e $49 \%$ da população caucasiana. Segundo Crump et al. (2001), estudos envolvendo populações caucasianas NorteAmericanas mostram que a ausência do gene GSTM-1 ocorre em cerca de $48-57 \%$ dos indivíduos analisados. Diversos estudos descritos na literatura mostram a freqüência deste gene em populações-controle de diferentes localidades e países (Tabela 1).

Apesar da freqüência do genótipo nulo em negros variar em torno de 20 a 31\% (LIN et al., 1994; LONDON et al., 1995; FORD et al., 2000) nas diferentes populações estudadas, as mais altas freqüências para o genótipo GSTM-1 nulo descritas na literatura (88$100 \%$ ) são referentes a estudos realizados com indivíduos do Pacífico Sul, que também possuem origem negróide (LIN et al., 1994; COTTON et al., 1999). Isto provavelmente se deve ao fato de que estas populações permaneceram isoladas durante um longo período, sob os efeitos da endogamia e da deriva genética.

Tabela 1 - Freqüência do genótipo GSTM-1 nulo em diversas populações-controle estudadas.

Tabela 2 - Freqüências do genótipo GSTM-1 nulo em populações-brasileiras controle. 
A Tabela 2 apresenta as freqüências obtidas para o polimorfismo do gene GSTM-1 em indivíduos brasileiros saudáveis de diferentes origens étnicas. A origem étnica da população brasileira é extremamente heterogênea, já que é composta de imigrantes europeus, africanos, asiáticos e índios; além disso, poucos estudos envolvendo o gene GSTM-1 em populações-controle têm sido realizados no Brasil, como os de Rossit et al. (1999), Hatagima et al. (2000), Gattás e Soares-Vieira (2000), D’Arce e Cólus (2000) e Losi-Guembarovski (2001), que analisaram a ausência do gene em indivíduos brasileiros saudáveis de diferentes origens étnicas. Os três primeiros estudos foram realizados com indivíduos provenientes da região Sudeste do Brasil (estados de São Paulo e Rio de Janeiro) e os dois últimos da região Sul do país (estado do Paraná) e encontraram, respectivamente, as freqüências de $44,6 \%, 46 \%, 54,8 \%, 33 \%$ e $48,86 \%$ de ausência para o gene GSTM-1. Arruda et al. (1998) estudaram este mesmo genótipo em brasileiros provenientes de três regiões distintas do país: Norte, Nordeste e Sudeste, encontrando a freqüência de $36,9 \%$. A maior prevalência para o genótipo nulo observada pelos autores ocorreu entre os caucasianos estudados $(55 \%)$, seguida pelos negros $(33 \%)$ e indígenas (20\%).

Losi-Guembarovski (2001) encontrou 47,88\% de ausência para este gene em sua amostra de caucasianos do Sul do Brasil, enquanto Gattás e Soares-Vieira (2000) estudando uma população da cidade de São Paulo encontraram a freqüência de $60,2 \%$ de ausência do gene GSTM-1 para esta mesma etnia. A mistura étnica, entretanto, é muito grande no Brasil, dificultando muitas vezes a determinação correta do grupo a que o indivíduo pertence e, conseqüentemente, a correlação entre determinados polimorfismos genéticos e etnia.

Estas variações na expressão do gene GSTM-1 em diferentes populações-controle leva a uma dificuldade em se selecionar grupos apropriados para a determinação de uma associação entre o genótipo GST e a susceptibilidade a doenças (HAYES; PULFORD, 1995) e para a extrapolação de resultados entre as diferentes populações (RAUNIO et al., 1995).

Os diferentes estudos desenvolvidos, não somente no Brasil, mas em diversos países, com genes responsáveis pela metabolização de xenobióticos, são de grande importância para o estabelecimento definitivo das freqüências destes genes nas diferentes populações humanas, e poderão futuramente ser utilizados como biomarcadores no auxílio à prevenção e tratamento do câncer.

\section{Agradecimentos}

As autoras agradecem a Srta. Liliane Moreira Nunes pela cuidadosa leitura e revisão do Abstract e à CAPES pelos recursos destinados à dissertação de mestrado.

\section{Referências}

ALEXANDRIE, A. et al. Genetic susceptibility to lung cancer with special emphasis on CYP1A1 and GSTM1: a study on host factors in relation to age at onset, gender and histological cancer types. Carcinogenesis, v.15, p.1785-1790, 1994.

ARRUDA, C.V.R. et al. Prevalence of homozygosity for the deleted alleles of glutathione S-transferase mu (GSTM1) and theta (GSTT1) among distinct ethnic groups from Brazil: relevance to enviromental carcinogenesis? Clinical Genetics, v.54, p.210-214, 1998.

AU, W.W. et al. Inheritance of polymorphic metabolizing genes and environmental disease and quality of life. Mutation Research, v.428, p.131-140, 1999.

BROCKMÖLLER, J. et al. Genotype and phenotype of glutathione S-transferase class $\mu$ isoenzymes $\mu$ and $\psi$ in lung cancer patients and controls. Cancer Research, v.53, p.1004-1001, 1993.

BUIM, M.E. Fatores genéticos na resposta a exposição ocupacional de agricultores paranaenses a agroquímicos. 1999. Dissertação (Mestrado em Genética e Melhoramento) - Universidade Estadual de Londrina, Londrina, 1999.

CARSTENSEN, U. et al. and T- lymphocyte micronulei in chimney sweeps with respect to genetic polymorphism for CYP1A1 and GST1 (class Mu). Mutation Research, v.289, p.187-195, 1993.

CAVENEE, W.K.; WHITE, R.L. The Genetic bases of cancer. An accumulation of genetic defects can apparently cause normal cells to become cancerous and cancerous cells to become increasingly dangerous. Scientific American, p.50-57, 1995.

CHEN, C. et al. Glutathione S-transferase M1 genotypes and the risk of squamous carcinoma of the cervix: a population-based case-control study. American Journal of Epidemiology, v.150, p.568-572, 1999.

CONDE, A.R. et al. Association of P53 glutathione Stransferase null genotype in gastric cancer in Portuguese population. Clinical Pathology: Molecular Pathology, v.52, p.131-134, 1999.

CONFORTI-FROES, N. et al. Predisposing genes and increased chromossome aberrations in lung cancer cigarrete smokers. Mutation Research, v.379, p.5359, 1997.

COTTON, S.C.; SHARP, L.J.; BROCKTON, N. Glutathione $S$ transferase polymorphisms and colorectal cancer. American Journal of Epidemiology, p.1-18, 1999.

CRUMP, C. et al. Glutathione S-transferase theta 1 gene deletion and risk of acute myeloid leukemia. Cancer Epidemiol Biomarkers Prev, v.9, p.457-460, 2000.

D'ARCE, L.P.G.; CÓLUS, I.M.S. Cytogenetic and molecular monitoring of brazilian farmers exposed to pesticides. Teratogenesis, Carcinogenesis and Mutagenesis, v.20, p.61-170, 2000.

DEAKIN, M. et al. Glutathione S-transferase GSTT1 genotypes and susceptibility to cancer studies of 
interactions with GSTM-1 in lung, oral, gastric and colorectal cancers. Carcinogenesis, v.17, p.881-884, 1996.

De JONG, J.L.; MOHANDAS, T.; TU, C.D. The human $\mathrm{H}$ (MU) class glutathione S-transferases are encoded by a dispersed gene family. Biochemical and Biophisycal Research Communications, v.180, p. 15-22, 1991.

FIGUEIRAS, J.T. et al. Glutathione S-transferase M1 (GSTM-1) and T1 (GSTT1) polymorphisms and lung cancer risk among Northwerstern Mediterraneans. Carcinogenesis, v.18, p.1529-1533, 1997.

FORD, J.G. et al. Glutathione S-transferase M1 polimorphism and lung cancer risk in AfricanAmericans. Carcinogenesis, v.21, p.1971-1975, 2000.

FRYER, A. A. et al. Use of site-directed mutagenesis of allele-specific PCR primers to identify the GSTM1 $A$, GSTM1 B, GSTM1 A, B, and GSTM1 null polymorphisms at the glutathione S-transferase, GSTM1 locus. Biochemical Journal, v.295, p. 313-315, 1993.

GATTÁS, G.J.F.; SOARES-VIEIRA, J.A. Cytochrome P450-2E1 and glutathione S-transferase polymorphisms among Caucasians and mulattoes from Brazil. Occupational Medicine, v.50, p.508-511, 2000.

GAWRONSKA, B. et al. CYP2D6 and GSTM1 genotypes in a Polish population. European Journal Clinical Pharmacologycal, v.55, p.389-392, 1999.

GEISLER, A.S.; OLSHAN, A. GSTM1, GSTT1 and the risk of Squamous Cell Carcinoma of the Head and Neck. American Journal of Epidemiology, v.154, p.95105, 2001.

HARADA, S. et al. Liver glutathione S-transferase polymorphism in Japanese and its pharmacogenetic importance. Human Genetics, v.75, p.322-325, 1987.

HATAGIMA, A. et al. Glutathione S-transferase M1 (GSTM-1) polymorphism in two Brazilian populations. Genetics and Molecular Biology, v.23, p.709-713, 2000.

HAYASHI, S.; WATANABE, J.; KAWAJIRI, K. High susceptibility to lung cancer analysed in terms of combined genotypes of P4501A1 and M $\mu$ class glutathione S-transferase genes. Japanese Journal Cancer Research, v.83, p.866-870, 1992.

HAYES, J.D.; PULFORD, D.J. The Glutathione Stransferase supergene family: regulation of $\mathrm{GST}^{*}$ and the contribuition of the isoenzymes to cancer chemoprotetion and drug resistance. Critical Reviews in Biochemistry and Molecular Biology, v.30, p. 445-600, 1995.

HIRVONEN, A et al. The GSTM1 null genotype as a risk modifier for squamous cell carcinoma of the lung. Carcinogenesis, v.14, p.1479-1482, 1993.

HIRVONEN, A. Genetic factors in individual responses to environmental exposures. Journal of Occupational and Environmental Medicine, v.37, p.37-41, 1995.

JOHNS, L.E.; HOULSTON, R.S. Glutathione Stransferase $\mu 1$ (GSTM1) status and bladder cancer risk: a meta-analysis. Mutagenesis, v.15, p.399-404, 2000.
KALANT, H. Biotransformações das drogas. In: KALANT, K.; ROSCHLAU, W. H. E.; SILVA, P. Princípios de Farmacologia Médica. Rio de Janeiro: Guanabara Koogan, 1991. p.30-40.

KETTERER, B et al. The human glutathione Stransferase supergene family, its polymorphism, and its effects on susceptibility to lung cancer. Environmental Health Perspectives, v.98, p.87-94, 1992.

KIHARA, M.; KIHARA, M.; NODA, K. Lung cancer risk of GSTM-1 null genotype in dependent on the extent of tabaco smoke exposure. Carcinogenesis, v.15, p.415-418, 1994.

KIHARA, M.; KIHARA, M.; NODA, K. Risk of smoking for squamous and small cell carcinomas of the lung modulated by combinations of CYP1A1 and GSTM1 gene polymorphisms in a Japanese population. Carcinogenesis, v.16, p.2331-2336, 1995.

KISS, I et al. Colorectal cancer risk in relation to genetic polymorphism of cytochrome P450 1A1, 2E1, and glutathione-S-transferase M1 enzymes. Anticancer Research, v.20, p.519-522, 2000.

LIN, H.J et al. Ethinic distribuition of the glutathione transferase MU (GSTM-1) null genotype in 1473 individuals and application to bladder cancer susceptibility. Carcinogenesis, v.15, p.1077-1081, 1994.

LONDON, S.J et al. Polymorphysm of glutathione Stransferase M1 and lung cancer risk among AfricanAmerican and Caucasians in Los Angeles County, Califórnia. Journal of the National Cancer Institute, v. 87, p.1246-1253, 1995.

LOSI-GUEMBAROVSKI, R. Estimativa da freqüência da indivíduos portadores do gene GSTM-1 na Região Norte do Paraná. 2001. Dissertação (Mestrado em Genética e Melhoramento) - Universidade Estadual de Londrina, Londrina 2001.

McWILLIAMS, J.E et al. Glutatione S-transferase M1 (GSTM1) deficiency and lung cancer risk. Cancer Epidemiology, Biomarkers \& Prevention, v.4, p.589-594, 1995.

NAKAGIMA, T et al. Expression and polymorphism of Glutathione S-transferase in human lungs: risk factors in smoking-related lung cancer. Carcinogenesis, v.16, p.707-711, 1995.

NEBERT, D.W. Role of genetics and drug metabolism in human cancer risk. Mutation Research, v.247, p.267281, 1991.

PALLI, D et al. Diet, metabolic polymorphisms and DNA adducts; the EPIC-Italy cross-sectional study. International Journal of Cancer, v.87, p.444-451, 2000.

PARKE, D.V. Activation mechanisms to chemical toxicology. Review Archieves of Toxicology, v.60, p.5$15,1987$.

PEARSON, W.R et al. Identification of class-mu glutathione transferase genes GSTMI-GSTM5 on human chromosome 1p13. American Journal of Human Genetics, v.53, p.220-233, 1993. 
RAUNIO, $\mathrm{H}$ et al. Diagnosis of polymorphims in carcinogen-activating and inactivating enzymes and cancer susceptibility - a review. Gene, v.159, p.113-121, 1995.

ROSS, V.L.; BOARD, P.G.; WEBB, G.C. Chromosomal mapping of the human mu class glutathione $S$ transferases to $1 \mathrm{p} 13$. Genomics, v.18, p.87-91, 1993.

ROSSIT, A.R.B.; CABRAL, I.R.; CONFORTI-FROES, N.D.T. Avaliação das freqüências alélicas de genes do biometabolismo em uma população brasileira. Genetics and Molecular Biology, v.22 (Suppl.), 1999.

RYBERG, D et al. Genotypes of glutathione transferase $\mathrm{M} 1$ and $\mathrm{P} 1$ and their significance for lung DNA adduct levels and cancer risk. Carcinogenesis, v.18, p.12851289, 1997.

SANTELLA, R.M et al. Policiclic aromatic hydrocarbonDNA and protein adducts in coal tar treated patients and controls and their relationship to gluthatione S-transferase genotype. Mutation Research, v.334, p.227-235, 1995.

SATO, $\mathrm{M}$ et al. Genetic polymorphism of drug metabolizing enzymes and susceptibility to oral cancer. Carcinogenesis, v.20, p.1927-1931, 1999.

SHIELDS, P.G et al. Polycyclic aromatic hydrocarbonDNA addusts in human lung cancer and susceptibility genes. Cancer Research, v.53, p.3486-3492, 1993.

SHIELDS, P.G. Pharmacogenetics: detecting sensitive populations. Environmental Health Perspectives, v.11 (Suppl.), p.81-87, 1994.

SEIDEGARD, $\mathrm{J}$ et al. Isoenzyme (s) of glutathione transferase (class $\mathrm{Mu}$ ) as a marker for the susceptibility to lung cancer: a follow up study. Carcinogenesis, v.11, p.33-36, 1990.
STRANGE, R.C et al. The human glutathione Stransferase: a case-control study of the incidence of the GST1 genotype in patients with adenocarcinoma. Carcinogenesis, v.12, p.25-28, 1991.

STÜCKER, I et al. GSTM1, smoking and lung cancer: a case control study. International Journal of Epidemiology, v.28, p.829-835, 1999.

VAN POPPEL, G. et al. Increased cytogenetic damage in smokers deficient in glutathiona S-transferase isozyme $\mu$. Carcinogenesis, v.13, p.303-305, 1992.

VENITT, S. Mechanisms of carcinogenesis and individual susceptibility to cancer. Clinical Chemistry, v.40, p.1421-1425, 1994.

VESELL, E.S. Genetic and environmental factors causing variation in drug response. Mutation Research, v.247, p.241-257, 1991.

ZHONG, S.; WOLF, C.R.; SPURR, N.K. Chromosomal assignment and linkage analysis of the human glutathione S-transferase $\mu$ gene (GSTM1) using intron specific polymerase chain reaction. Human Genetics, v.90, p.435-439, 1992.

ZHONG, S et al. Relationship between the GSTM1 genetic polymorphism and susceptibility to bladder, breast and colon cancer. Carcinogenesis, v.14, p.18211824, 1993a.

ZHONG, $S$ et al. Deduced amino acid sequence, gene structure and chromosomal location of a novel human class $\mathrm{Mu}$ glutatione S-transferase, GSTM4. Biochemical Journal, v.291, p.41-50, 1993b. 